INNATE IMMUNITY

\section{Reigning in RIG-I}

Tempering type I interferon (IFN) responses triggered by viral infection is thought to be important to minimize collateral tissue damage. Now, researchers have identified a long non-coding RNA (lncRNA) that is induced in host cells after viral infection and inhibits late-stage type I IFN responses. It works by binding to and inactivating the viral RNA sensor retinoic acid-inducible gene I (RIG-I).

By performing immunoprecipitation assays using a mouse macrophage cell line that was either uninfected or infected with the RNA virus vesicular stomatitis virus (VSV), the authors identified numerous RIG-I-interacting host RNAs. These RNAs were ranked according to abundance and the 30 most abundant were knocked down using small interfering RNAs to identify those with functional effects. Knockdown of one particular lncRNA - termed lnc-Lsm3b - led to a marked increase in VSV-induced type I IFN production, suggesting a role in the regulation of antiviral responses.
More detailed analysis showed that the expression of lnc-Lsm3b was induced by RNA-virus infection or by prolonged stimulation with high concentrations of type I IFNs. lnc-Lsm3b expression was completely abolished in macrophages that lacked the type I IFN receptor, suggesting that it is an IFN-stimulated gene. Pull-down assays indicated that lnc-Lsm $3 b$ binding to RIG-I was most pronounced $8 \mathrm{hr}$ and $12 \mathrm{hr}$ after viral infection.

To explore the function of lnc-Lsm3b further, the authors analysed a lnc-Lsm3b-deficient macrophage line. Following RNA-virus challenge,

lnc-Lsm3b-deficient cells produced higher levels of type I IFNs and, accordingly, showed reduced viral replication than control cells. Similarly, lnc-Lsm3b-deficient mice were less susceptible to VSV-induced lethality than wild-type mice, owing to higher serum levels of type I IFNs. lnc-Lsm3b-deficient mice also showed better control of other RNA-virus infections, but not of DNA-virus infection,

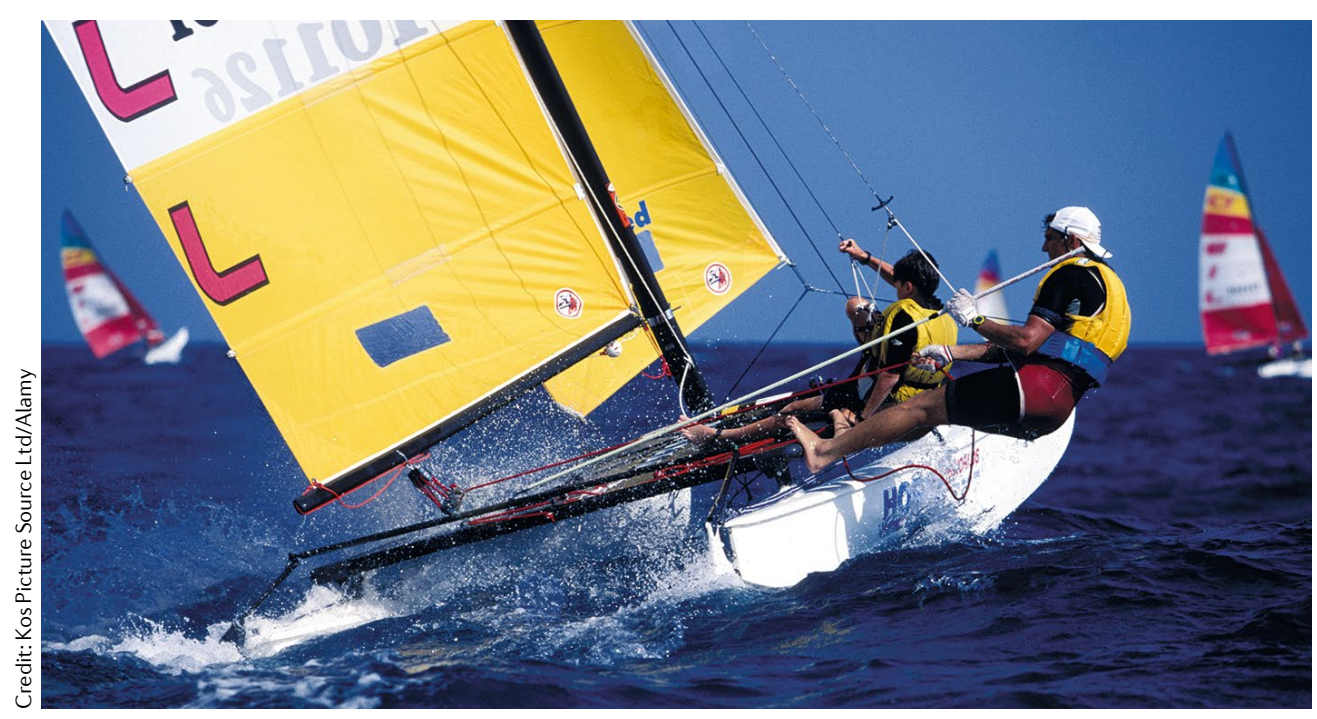

indicating selective inhibition of RIG-I-mediated antiviral responses.

In terms of the mechanism of action of lnc-Lsm3b, the authors showed that RIG-I inhibition did not involve reduced RIG-I protein expression but involved interrupted RIG-I signalling capability. lnc-Lsm3b was shown to bind to the C-terminal domain of RIG-I and, importantly, binding of lnc-Lsm3b to RIG-I outcompeted binding by viral RNA. A GA-rich sequence of lnc-Lsm3b that forms a double-stranded RNA stem-loop structure was required for its high affinity binding to and inhibition of RIG-I.

Viral infection induces the formation of RIG-I oligomers; lnc-Lsm3b inhibited this oligomerization by binding to and sequestering RIG-I monomers. Specifically, lnc-Lsm3b stabilized the interaction between the RIG-I caspase recruitment domain (CARD) and helicase domain, keeping it in an auto-repressed conformation. Transition to activated RIG-I requires ATP binding and hydrolysis. lnc-Lsm3b effectively suppressed viral RNA-induced ATPase activity of RIG-I, thereby maintaining RIG-I in an inactive state. Finally, the authors confirmed that lnc-Lsm3b inhibits downstream signalling of RIG-I by showing that lnc-Lsm3b-deficient cells had increased interactions between RIG-I and its downstream signalling mediators mitochondrial antiviral-signalling protein (MAVS) and TRIM25.

So, this study shows that recognition of an inducible self-RNA by RIG-I can terminate late antiviral immune responses in a feedback manner. By efficiently competing with viral RNAs for binding to RIG-I, lnc-Lsm3b acts as a decoy to temper the IFN response.

Lucy Bird

ORIGINAL ARTICLE Jiang, M. et al.

Self-recognition of an inducible host lncRNA by RIG-I feedback restricts innate immune response. Cell. 173, 906-919 (2018) 